FreUnd, A. (1973). PhD Thesis, Technische Universität München.

FreUnd, A. (1975a). Anomalous Scattering, Edited by S. Ramaseshan \& S. C. Abrahams, pp. 69-84. Copenhagen: Munksgaard.

FrEUND, A. (1975b). Nucl. Instrum. Meth. 124, 93-99.

FREUND, A. (1976). Progress in Neutron Monochromator Development at the Institut Laue-Langevin. Proceedings of the Gatlinburg Conference, June 1976, USA.

Freund, A. \& Schnemer, J. R. (1972). J. Cryst. Growth, 13/14, 247-251.

HoHlWein, D. (1975). J. Appl. Cryst. 8, 465-468.

Hosoya, S. \& Yamagishi, T. (1966). J. Phys. Soc. Japan, 21, 2638-2644.

Jennings, L. D., Chipman, D. R. \& DeMarco, J. J. (1964). Phys. Rev. 135, 1612-1615.

Larson, A. C. (1967). Acta Cryst. 23, 664.
Lehmann, M. S. \& Larsen, F. K. (1974). Acta Cryst. A30, 580-584.

Neutron Cross Sections (1955). BNL Report 325, Brookhaven National Laboratory, Upton, NY 11973, USA.

SCHNEIDER, J. R. (1974a). J. Appl. Cryst. 7, 541-546.

SCHNEIDER, J. R. (1974b). J. Appl. Cryst. 7, 547-554.

SCHNEIDER, J. R. (1975a). J. Appl. Cryst. 8, 195-201.

SCHNEIDER, J. R. (1975b). J. Appl. Cryst. 8, 530-534.

SCHNEIDER, J. R. (1976). J. Appl. Cryst. 9, $394-402$.

SCHNEIDER, J. R. (1977). Acta Cryst. A 33, 235-243.

SEeger, A. A. (1965). Editor, Moderne Probleme der Metallphysik. Vol. 1. Heidelberg: Springer Verlag.

Temkin, R. J., Henrich, V. E. \& RaccaH, P. M. (1972). Phys. Rev. B6, 3572-3581.

Thornley, F. R. \& Nelmes, R. J. (1976). Acta Cryst. A30, 748-757.

Zachariasen, W. H. (1967). Acta Cryst. 23, 558-564.

Acta Cryst. (1977). A33, 800-804

\title{
A Structure-Factor Least-Squares Refinement Procedure for Macromolecular Structures using Constrained and Restrained Parameters
}

\author{
By Joel L. Sussman, ${ }^{*}$ Stephen R. Holbrook, George M. Church and Sung-Hou Kim \\ Department of Biochemistry, Duke University Medical Center, Durham, North Carolina 27710, USA
}

(Received 22 February 1977; accepted 18 April 1977)

\begin{abstract}
A method of structure-factor least-squares refinement of constrained groups linked by distance restraints has been developed for the refinement of macromolecular structures. Each constrained group can have any number of variable dihedral rotation parameters within the group in addition to the rigid-body translational, rotational and thermal parameters. The matrix of normal equations may be either full or sparse and provision is made for solution by matrix inversion or the conjugate-gradient iterative method. This procedure has been successfully used for $3 \AA$ data and should be applicable even for lower-resolution data and especially for cases with a poorer data-per-atom ratio. The structure of yeast phenylalanine tRNA has been refined with this procedure from a starting crystallographic $R$ value of $42 \%$ to a final $R$ value of $25 \%$ with isotropic 'group' thermal parameters and $22 \%$ with isotropic atomic thermal parameters for 8207 independent reflections at $2.7 \AA$ resolution. The proper stereochemistry of bond distances, angles and van der Waals contacts for the restrained atoms was maintained within reasonable limits throughout the refinement. Although originally developed for nucleic acids, this procedure is directly applicable to the refinement of protein structures. In addition, a combination of applying distance restraints between groups and least-squares fitting of these groups to target coordinates has been used purely as an idealization process for imposing proper stereochemistry on an approximate model.
\end{abstract}

\section{Introduction}

As the number of macromolecules for which an approximate model has been derived by $\mathrm{X}$-ray diffraction methods has increased, several methods of structure refinement have been tried.

To refine protein structures in real space, Freer, Alden, Carter \& Kraut (1975) automated the difference Fourier technique of obtaining shifts, and introduced differential methods of calculating the slope of the electron density at the grid points in conjunction with imposition of restraints between cycles. Diamond

\footnotetext{
* Present address: Department of Structural Chemistry, The Weizmann Institute of Science, Rehovot, Israel.
}

(1971) has devised a procedure for minimizing the difference between an electron density map and a model density map, calculated assuming a Gaussian distribution of electron densities centered at the atomic positions from the current model, by changing only torsional angles and some bond angles of the constrained model. Among others, Deisenhofer \& Steigemann (1975) applied this procedure together with Fourier maps calculated with new phases after each cycle to refine the structure of the pancreatic trypsin inhibitor.

The structure refinement of macromolecules in reciprocal space was pioneered by Watenpaugh, Sieker, Herriot \& Jensen (1973), who showed that it was possible to refine a protein structure by a least-squares 
method on the individual atomic parameters. They successfully lowered the residual $(R)$ from $22 \cdot 4$ to $12 \cdot 6 \%$ for rubredoxin and improved the model and map using 5005 reflections for 558 non-hydrogen atoms. Recently, Konnert (1976a) has improved this approach by introducing subsidiary distance restraints as described by Waser (1963) to maintain proper stereochemistry throughout refinement and ensure convergence with a limited subset of data. He reports successfully refining the 2436 atomic positional parameters (812 atoms) of carp calcium binding protein using only 1370 intensity data by including 2030 distance restraints. Three other approaches which are directed to improvement of the phases, and therefore, the interpretability of the electron density map have been developed by Hendrickson \& Karle (1973), Sayre (1974), and Collins, Cotton, Hazen, Meyer \& Morimoto (1975).

With a few exceptions, X-ray diffraction data from protein and nucleic acid crystals do not warrant the conventional structure-factor least-squares refinement where individual atomic positions are independently refined. Meaningless shifts of atomic positions result from such refinement, primarily as a result of the low data/parameter ratio and the relatively poor quality of the data compared to most of the small molecule X-ray data. To improve the situation, constrained group refinement was attempted in the early work on myoglobin (Brändén, Holmes \& Kendrew, 1963) and in refinement of models using fibre diffraction data (Arnott, Dover \& Wonnacott, 1969). Such approaches are justifiable because proteins and nucleic acids can be considered as composed of many 'rigid' groups; peptide groups, as a first approximation, many side chains (phenyl, tyrosyl, tryptophanyl, prolyl, valyl, etc.) or parts of side chains in proteins; bases, phosphates and riboses in nucleic acids.

To take advantage of the rigid groups in proteins and nucleic acids, to overcome the relatively poor quality and resolution of the data from such crystals, and to reduce the influence of the linked neighbors on positional shift, we developed a scheme of structure factor refinement which combines Scheringer's rigidgroup constraints (Scheringer, 1963a), as extended to allow for variable rotation axes (Scheringer, 1963b), with distance restraints (Waser, 1963) to maintain proper stereochemistry between groups within a specified error limit. The advantages of a constrained group refinement (Scheringer, 1963a) are (1) a large increase in the data/parameters ratio: (2) automatic maintenance of group stereochemistry; (3) increased range of convergence; (4) reduced computing time and core storage resulting from a smaller normal equations matrix; (5) applicability of the method to intermediate resolution data. By allowing variable dihedral angles within constrained groups it is possible to choose the groups in such a manner that the total number of structural parameters is further reduced.

Distance restraints are applied between any two atoms of the same or different groups in order to maintain the appropriate stereochemistry and effectively further reduce the degrees of freedom of the system. In addition to maintaining proper bond lengths and angles at connections between rigid groups, we have also used distance restraints to minimize bad nonbonded contacts. A list of minimum non-bonded contacts is prepared and whenever a group is shifted by refinement to within the extreme close contact distance specified (Ramachandran \& Sasisekharan, 1968), appropriately weighted restraint terms are added into the least-squares matrix which tend to shift the group parameters in such a way as to alleviate the bad contact. This approach is similar to a hard-sphere potential frequently used in energy calculation.

In addition to application in structure-factor refinement, distance restraints can be used for stereochemical idealization of a model structure (Waser, 1963). The usual procedure has been to restrain all bond distances, bond angles and any fixed torsion angles to specified 'ideal' values (Levitt \& Lifson, 1969; Hermans \& McQueen, 1974; Dodson, Isaacs \& Rollett, 1976). We have, however, followed an approach consistent with our structure-factor refinement. The distances between the atomic coordinates of the constrained groups and the target coordinates of the model are minimized, and at the same time, the intergroup connections are restrained. Since the only restraints are at the links between groups, and since all shifts involve only the group translational and rotational parameters, far fewer parameters and restraints are required than when using restraints between atoms. We have used this method for idealizing a nucleic acid model and it is directly applicable to model building of protein structures.

\section{Least-squares refinement using constraints and restraints}

The quantity to be minimized, $Q$, in the least-squares procedure consists of the sum of three terms:

$$
Q=w_{F} \mathrm{DF}+w_{D} \mathrm{DD}+w_{T} \mathrm{DT}
$$

where $w_{F}, w_{D}$, and $w_{T}$ are overall weights for each term. The first term, DF, is the usual structure-factor differences summed over all or part of the reflections, $h$ :

$$
\mathrm{DF}=\sum_{h} w_{h}\left(\left|\mathbf{F}_{o, h}\right|-\left|\mathbf{F}_{c, h}\right|\right)^{2}
$$

The second term restrains the stereochemistry and is the sum over all subsidiary distance restraints, $d$ :

$$
\mathrm{DD}=\sum_{d} w_{d}\left(D_{o, d}-D_{c, d}\right)^{2},
$$

where $D_{o, d}$ is the 'ideal' distance between specified pairs of atoms (which may correspond to a bond length, bond angle or a non-bonded close-contact distance) and $D_{c, d}$ is this distance calculated from the model. The third term restrains the structure from moving away from a specified set of target coordinates. Here the sum 
is over all atoms, $i$, and over three axial components, $j$, of each atom:

$$
\mathrm{DT}=\sum_{i} w_{i} \sum_{j}\left(X_{T, i, j}-X_{i, j}\right)^{2},
$$

where $X_{T, i, j}$ is the axial coordinate (orthogonal and in $\AA$ ) of the target atom, while $X_{i, j}$ is the corresponding coordinate of the model.

For restrained-constrained structure-factor leastsquares refinement, we set $w_{T}=0$, while for distancetarget idealization (model building), $w_{F}=0$. The relative magnitudes among $w_{F}, w_{D}$ and $w_{T}$ have been discussed earlier (Waser, 1963; Konnert, 1976a).

All three of the above terms are functions of the atomic coordinates of the model structure. Starting from a set of coordinates for a group of atoms in $\AA, \mathbf{X}$, if one assumes six rigid-body parameters (three rotational and three translational) and an arbitrary number of dihedral-angle parameters, one can convert to fractional coordinates, $\mathbf{x}$, with the following expression:

$$
\mathbf{x}=\mathbf{t}_{\mathrm{g}}+[\mathbf{U}]\left[\mathbf{R}_{\mathrm{g}}\right]\left[\mathbf{D}_{\mathrm{g}, \psi_{1}}\right] \ldots\left[\mathbf{D}_{\mathrm{g}, \psi_{\mathrm{k}}}\right] \mathbf{X},
$$

where: $\mathbf{t}_{\mathrm{g}}$ is the translation vector $(x, y, z)$ in fractional coordinates of the origin of the group, $g$, (usually taken at or near its center of mass) relative to the origin of the unit cell; $[\mathbf{U}]$ is the matrix which transforms Cartesian coordinates in $\AA$ to fractional crystallographic coordinates (Rossman \& Blow, 1962)

$$
[\mathbf{U}]=\left(\begin{array}{llr}
1 /(a \sin \gamma \sin \omega) & 0 & 0 \\
1 /(b \tan \alpha \tan \omega)-1 /(b \tan \gamma \sin \omega) & 1 / b & -1 /(b \tan \alpha) \\
-1 /(c \sin \alpha \tan \omega) & 0 & 1 /(c \sin \alpha)
\end{array}\right),
$$

where $a, b, c, \alpha, \beta, \gamma$, are the unit-cell parameters, and

$$
\cos \omega=(\cos \beta-\cos \alpha \cos \gamma) /(\sin \alpha \sin \gamma) ;
$$

$\left[\mathbf{R}_{g}\right]$ is the rotation matrix (Doedens, 1970) corresponding to successive rotations of the entire group, first, about the $z$ axis by $\varphi$ degrees, next, about the new $x$ axis by $\theta$ degrees, and finally about the new $y$ axis by $\varrho$ degrees,

$$
\left[\mathbf{R}_{\mathrm{g}}\right]=\left(\begin{array}{ccc}
\cos \varphi \cos \varrho-\sin \varphi \sin \theta \sin \varrho & -\sin \varphi \cos \theta & \cos \varphi \sin \varrho+\sin \varphi \sin \theta \cos \varrho \\
\sin \varphi \cos \varrho+\cos \varphi \sin \theta \sin \varrho & \cos \varphi \cos \theta & \sin \varphi \sin \varrho-\cos \varphi \sin \theta \cos \varrho \\
-\cos \theta \sin \varrho & \sin \theta & \cos \theta \cos \varrho
\end{array}\right) ;
$$

and $\left[\mathbf{D}_{g, \psi k}\right]$ is the dihedral rotation matrix (the transpose of the matrix as given by Patterson, 1959), for a particular dihedral angle of group $g$, which is applied to a 'subgroup' of the atoms in the group, e.g. rotation of a sugar about the glycosyl bond relative to a base, where $\psi_{k}$ is the amount of rotation of the $k$ th dihedral angle, and $l_{1,2,3}$ are the direction cosines of the particular rotation axis with respect to the Cartesian coordinate system.

$$
\left[\mathbf{D}_{g, \psi_{k}}\right]=\left(\begin{array}{lll}
\cos \psi_{k}+l_{1}^{2}\left(1-\cos \psi_{k}\right) & l_{1} l_{2}\left(1-\cos \psi_{k}\right)-l_{3} \sin \psi_{k} & l_{3} l_{1}\left(1-\cos \psi_{k}\right)+l_{2} \sin \psi_{k} \\
l_{1} l_{2}\left(1-\cos \psi_{k}\right)+l_{3} \sin \psi_{k} & \cos \psi_{k}+l_{2}^{2}\left(1-\cos \psi_{k}\right) & l_{2} l_{3}\left(1-\cos \psi_{k}\right)-l_{1} \sin \psi_{k} \\
l_{3} l_{1}\left(1-\cos \psi_{k}\right)-l_{2} \sin \psi_{k} & l_{2} l_{3}\left(1-\cos \psi_{k}\right)+l_{1} \sin \psi_{k} & \cos \psi_{k}+l_{3}^{2}\left(1-\cos \psi_{k}\right)
\end{array}\right) .
$$

To return to orthogonal $\AA$ coordinates from fractional coordinates, as is required in DD and DT above, one merely multiplies the fractional coordinates by the inverse of $[\mathbf{U}]$ :

$$
[\mathbf{U}]^{-1}=\left(\begin{array}{lll}
a \sin \gamma \sin \omega & 0 & 0 \\
a \cos \gamma & b & c \cos \alpha \\
a \sin \gamma \cos \omega & 0 & c \sin \alpha
\end{array}\right)
$$

$$
\begin{gathered}
{[\mathbf{A}] \Delta \mathbf{p}=\mathbf{V}} \\
A_{n m}=w_{F} \sum_{h} w_{h} \frac{\partial\left|\mathbf{F}_{c, h}\right|}{\partial P_{n}} \frac{\partial\left|\mathbf{F}_{c, h}\right|}{\partial P_{m}}+w_{D} \sum_{d} w_{d} \frac{\partial D_{c, d}}{\partial P_{n}} \frac{\partial D_{c, d}}{\partial P_{m}} \\
+w_{T} \sum_{i} w_{i} \sum_{j} \frac{\partial X_{i, j}}{\partial P_{n}} \frac{\partial X_{i, j}}{\partial P_{m}}
\end{gathered}
$$




$$
\begin{aligned}
V_{n} & =w_{T} \sum_{h} w_{h}\left(\left|\mathbf{F}_{o, h}\right|-\left|\mathbf{F}_{c, h}\right|\right) \frac{\partial\left|\mathbf{F}_{c, h}\right|}{\partial P_{n}} \\
& +w_{D} \sum_{d} w_{d}\left(D_{o, d}-D_{c, d}\right) \frac{\partial D_{c, d}}{\partial P_{n}} \\
& +w_{T} \sum_{i} w_{i} \sum_{j}\left(X_{T, i, j}-X_{i, j}\right) \frac{\partial X_{i, j}}{\partial P_{n}} .
\end{aligned}
$$

The contributions to the A matrix from the distance cross derivatives are non-zero only for elements $A_{n m}$, where $P_{n}$ and $P_{m}$ are both parameters of groups which are restrained to each other $\left(P_{n}\right.$ and $P_{m}$ affect $\left.D_{c, d}\right)$. When idealizing a model $\left(w_{F}=0, w_{T}>0\right)$, the matrix element contributions from the targeting cross derivatives are non-zero only when the Cartesian coordinate $X_{i, j}$ is a function of both parameters $P_{n}$ and $P_{m}$.

\section{Construction and solution of the normal-equation matrix}

Even though the number of parameters describing a macromolecule may be greatly decreased by employing rigid-body constraints, the use of full-matrix methods still may be prohibitive for many macromolecular structures in terms of storage space and time of matrix construction.

When this is the case, we have used a 'sparse matrix', which contains blocks of intragroup cross derivatives along the diagonal and off-diagonal blocks containing cross-derivative terms between groups sharing at least one restraint condition, including possible van der Waals contacts (whether within contact distance or not). This has the advantage of retaining many elements of the full matrix off the diagonal, which have a significant effect on the shifts of the group parameters. Because of the spatial proximity of restrained groups, the shifts of these groups are highly correlated. All other off-diagonal elements should have relatively small influence and are thus filled with zeros. Since the operations involved in filling the elements of a full matrix are so numerous (often more time consuming than matrix solution), a great saving in calculation time can be gained by use of a 'sparse matrix' where most of the terms are simply set to zero.

Such a 'sparse matrix' may be solved by either matrix inversion or an iterative procedure such as the conjugate-gradient method (Hestenes \& Stiefel, 1952). We have found that time spent in actual solution of the normal equations by the conjugate-gradient method is comparable to that used in matrix inversion by the Choleski procedure (for example, see Rollett, 1965).

Since the conjugate-gradient method requires storing only the non-zero elements, a saving in storage space of up to $99 \%$ may result. However, use of matrix inversion may at times be useful because of the ease of calculation of estimated standard deviations and correlation coefficients.

It should be noted that in order for the conjugategradient method to be successful in solving a matrix, all matrix elements should be of approximately the same magnitude. Since for example, the $\partial \mathbf{F}_{c} / \partial B$ and $\partial \mathbf{F}_{c} / \partial x$ may be very different, it is necessary to scale the elements of the matrix according to estimates of the average values of the various types of derivatives. This scaling is then reversed after the shifts have been calculated (Konnert, 1976b).

\section{Refinement of yeast phenylalanine tRNA}

The method of constrained-restrained structure-factor least squares discussed above was initially developed for refinement of the crystal structure of yeast phenylalanine tRNA. It was felt that a refinement with constraints and restraints was necessary, considering the limited resolution of the data and the resulting poor data/parameter ratio.

We have generated 'idealized' starting atomic coordinates of yeast phenylalanine tRNA by connecting the stereochemically ideal constrained groups with restrained distances, then simultaneously minimizing the distances between the coordinates of the ideal groups and the target coordinates. The target coordinates were from the model of the tRNA derived by the multiple isomorphous replacement and partial Fourier methods (Sussman \& Kim, 1976a).

In the early stages of refinement, only 6153 data between 3 and $10 \AA$ resolution were used in order to increase the radius of convergence of the least-squares procedure and to save calculation time. The 1652 atoms of tRNA were divided into 132 constrained groups. In addition, distance restraints were included which specified the stereochemistry of the phosphate-sugar connections, hydrogen bonds in the accepted secondary and tertiary interactions (Sussman \& Kim, 1976b), and hard-sphere contact distances of non-bonded atom pairs. These restraints, along with maintaining the proper stereochemistry, served to effectively improve the data/parameter ratio. At this point, there were 888 variable positional parameters representing the entire structure along with an overall temperature factor and scaling factors.

After four cycles of refinement for these parameters, we increased the resolution of the observations used to $2 \cdot 7 \AA$ (8207 reflections with $F>2 \sigma$ used out of 8579 measured), removed distance restraints for the tertiary $\mathrm{H}$ bonds, and allowed temperature factors to vary for each phosphate, ribose, or base group. We then introduced variable sugar pucker by defining three variable dihedral rotations for each sugar and simultaneously restraining distances between atoms within each ribose. The total number of positional parameters was thus increased to 1121 plus 228 variable subgroup (phosphates, riboses and bases) temperature factors. At this point, $88 \%$ of the total chemical bonds and $84 \%$ of the total bond angles in the structure were constrained and the remainders restrained. Three cycles of refinement on 1652 variable atomic thermal parameters concluded the refinement.

The complete refinement lowered the $R$ value from 
$42 \%$ for 6153 reflections ( $3 \AA$ data) to $25 \%$, with isotropic subgroup thermal parameters, then to $22 \%$, by refinement of just isotropic atomic thermal parameters, for 8207 reflections $(2 \cdot 7 \AA$ data). The metal ions, bound waters, hydrogens and solvent were not included in the refinement. The correlation coefficient between observed and calculated structure factors (Bevington, 1969 ) at this point was 0.87 . The average change in atomic coordinates from the starting model was $0.83 \AA$, the root mean square (r.m.s.) change was $1 \cdot 10 \AA$, and the average phase shift was $55^{\circ}$. The overall quality of the difference Fourier and Fourier maps was greatly improved. The difference Fourier and $2 F_{o}-F_{c}$ Fourier maps were calculated and observed on a computer graphics system at four different stages of the refinement, including the final one. This was very advantageous in manual correction of the model at points where a group had shifted to an incorrect local minimum because of poor starting coordinates.

The computing time for one cycle of constrainedrestrained least-squares refinement of the 888 variable parameters of tRNA ( 1652 atoms) using 6153 data was $33 \mathrm{~min}$ on an IBM $370 / 165$ machine. This includes $15 \mathrm{~min}$ of computing time required for structure-factor calculations alone.

All bond distances and angles within constrained groups (over $85 \%$ of total bond distances and angles in the structure) retained their canonical values. The average deviation from 'ideality' in the restraint distances corresponding to bond lengths was $0.02 \AA$ (r.m.s. deviation $=0.03 \AA$ ), the restraints corresponding to bond angles and dihedrals deviated by an average of $0.03 \AA$ (r.m.s. deviation $=0.04 \AA$ ), which corresponds to about $2^{\circ}$. The shortest non-bonded contact was $0 \cdot 3 \AA$ less than the closest allowed contact distance (Ramachandran \& Sasisekharan, 1968). The maximum deviation in restrained bond distance was $0.15 \AA$ and in restrained angle $0 \cdot 15 \AA$, which corresponds to about $10^{\circ}$.

Structural details and analysis of the application of this refinement technique on the yeast phenylalanine tRNA structure will be published elsewhere.

Note added in proof: This program has recently been applied successfully for model building and refinement of two protein structures: demetallized concanavalin A where the $R$ value was reduced from 47 to $28 \%$ ( $3.2 \AA$ data) and triclinic lysozyme where the $R$ value was reduced from 35.5 to $25.6 \%$ (2.5 $\AA$ data). In both cases after refinement the quality of the electron density maps was greatly improved while the models of the structures retained good stereochemistry (Sussman, unpublished).

This work was supported by the grants from NIH (CA 15802) and NSF (GB 40814).

\section{References}

Arnott, S., Dover, S. D. \& Wonacott, A. J. (1969). Acta Cryst. B 25, 2192-2206.

BEVINGTON, P. R. (1969). Data Reduction and Error Analysis for the Physical Sciences, p. 119. New York: McGrawHill.

BrändÉn, C.-I., Holmes, K. C. \& Kendrew, J. C. (1963). Acta Cryst. 16, A 175.

Collins, D. M., Cotton, F. A., Hazen, E. E. JR, Meyer, E. F. JR, \& MoRImoto, C. N. (1975). Science, 190, 10471052.

DiAmOND, R. (1971). Acta Cryst. A 27, 436-452.

Deisenhofer, J. \& SteigemanN, W. (1975). Acta Cryst. B31, 238-250.

Dodson, E. J., Isaacs, N. W. \& Rollett, J. S. (1976). Acta Cryst. A 32, 311-315.

DOEDENS, R. (1970). Crystallographic Computing, pp. 198200. Copenhagen: Munksgaard.

Freer, S. T., Alden, R. A., Carter, C. W. Jr \& Kraut, J. (1975). J. Biol. Chem. 250, 46-54.

HeNDRICKSON, W. A. \& KARLE, J. (1973). J. Biol. Chem. 248, 3327-3334.

Hermans, J. \& McQueen, J. E. JR (1974). Acta Cryst. A 30, 730-739.

Hestenes, M. R. \& Stiffel, E. (1952). J. Res. Natl. Bur. Stand. 49, 409-436.

KONNERT, J. H. (1976a). Acta Cryst. A 32, 614-617.

KONNERT, J. H. (1976b). Personal communication.

LeVitt, M. \& Lifson, S. (1969). J. Mol. Biol. 46, 269-279.

Patterson, A. L. (1959). International Tables for X-ray Crystallography, Vol. II, p. 63. Birmingham: Kynoch Press.

Ramachandran, G. N. \& Sasisekharan, V. (1968). Advanc. Protein Chem. 23, 283-437.

RolletT, J. S. (1965). Editor Computing Methods in Crystallography, p. 19. Oxford: Pergamon Press.

Rossmann, M. G. \& Blow, D. M. (1962). Acta Cryst. 15, 24-31.

SAYre, D. (1974). Acta Cryst. A 30, 180-184.

SCHERINGER, C. (1963a). Acta Cryst. 16, 546-550.

SCHERINGER, C. (1963b). Acta Cryst. 16, A 175.

Sussman, J. L. \& Kim, S. H. (1976a). Biochem. Biophys. Res. Commun. 68, 89-96.

Sussman, J. L. \& KIM, S. H., (1976b). Science, 192, 853-858.

WASER, J. (1963). Acta Cryst. 16, 1091-1094.

Watenpaugh, K. D., Sieker, L. C., Herriott, J. R. \& JeNSEN, L. H. (1973). Acta Cryst. B 29, 943-956. 